\title{
Lognormal variability in BL Lacertae (Research Note)
}

\author{
B. Giebels and B. Degrange
}

\author{
Laboratoire Leprince-Ringuet, École polytechnique, CNRS/IN2P3, 91128 Palaiseau, France \\ e-mail: berrie@poly.in2p3.fr
}

Received 8 April 2009 / Accepted 15 June 2009

\begin{abstract}
Context. The characterization of a time series is a powerful tool for investigating the nature of mechanisms that generate variability in astrophysical objects. Blazar variability across the entire electromagnetic spectrum is a long-standing puzzle, and it has been difficult to ascertain the mechanisms at play.

Aims. Lognormal variability in X-ray light curves, probably related to accretion disk activity, has been discovered in various compact systems, such as Seyfert galaxies and X-ray binaries. Identifying a similar behaviour in blazars would establish a link between them. Methods. Public X-ray data from the blazar BL Lac are used to investigate the nature of its variability, and more precisely the flux dependency of the variability and the distribution of fluxes.

Results. The variations in the flux are found to have a lognormal distribution and the average amplitude of variability is proportional to the flux level.

Conclusions. BL Lac is the first blazar in which lognormal X-ray variability is clearly detected. The light curve is orders of magnitude less variable than other blazars, with few bursting episodes. If this defines a specific state of the source, then the lognormality might be the imprint of the accretion disk on the jet, linking for the first time accretion and jet properties in a blazar.
\end{abstract}

Key words. X-rays: galaxies - galaxies: active - galaxies: BL Lacertae objects: individual : BL Lac radiation mechanisms: non-thermal

\section{Introduction}

The luminosity fluctuations from astronomical sources are often used as a probe to constrain the physical mechanisms that generate them. Nonthermal X-rays from blazars are understood to originate in relativistic electrons cooling either through the synchrotron or the inverse Compton mechanisms. Their spectra are usually featureless power law continuums, with little or no thermal emission from the disk surrounding the central black hole. If thermal emission is present, it is difficult to find because of the dominance of the nonthermal radiation (Perlman et al. 2008), complicating further the study of the link between accretion and jet physics in these objects. Analyses have attempted to identify in particular features in light curves where the flux varies significantly, such as peaks, interpreted as the light crossing time through an emission zone and hence a direct probe of its geometrical size. The cause of variability is still poorly understood despite decades of observations across the entire electromagnetic spectrum.

We analyze the light curve of BL Lacertae (BL Lac), a moderately X-ray bright blazar of the class named after itself. It has never shown dramatic X-ray outbursts, where fluxes vary by over an order of magnitude or more, as is often the case for this class of source when they are also very high energy $\gamma$-ray emitters ${ }^{1}$. The available data set has already provided a wealth of results, the most notable being the observation of a double X-ray flare in 2005, interpreted by Marscher et al. (2008) as originating from a disturbance passing through two different zones in the jet of BL Lac. The relatively major outburst-free light curve

${ }^{1}$ BL Lac's spectrum extends up to $\sim 1 \mathrm{TeV}$, see Albert et al. (2007). is probed for evidence of a statistical property found in some accreting sources, called lognormality, found in galactic as well as extragalactic accreting sources which can be generated by a stationary process by taking the exponential of a Gaussian time series (Uttley et al. 2005). However, McHardy (2008) suggested that variations generated within the accretion disk can modulate the nonthermal jet emission. Evidence of lognormal variability has also been found in very high-energy gamma-ray emission from the BL Lac object PKS 2155-304 (Degrange et al. 2008). Lognormal fluxes have fluctuations that are on average proportional to the flux itself, and are the signature of an underlying multiplicative physical process, rather than additive. This signature is particularly difficult to find in blazars because they are relatively faint, causing the Poisson noise to be large, and usually densely sampled only on flaring occasions and for a few days, but even then fluxes remain modest compared to those of $\mathrm{X}$-ray binaries.

\section{Data and analysis}

BL Lac has been observed routinely by the PCA detectors (Jahoda et al. 1995) onboard RXTE for many years, and we analyze $6.75 \times 10^{5} \mathrm{~s}$ of public data collected since March 2005, with exposures of a few ks per observation. This corresponds to the RXTE ObsID's numbered 91127,92103 and 93135 . The PCA data were analyzed using the HEASOFT 6.5.1 package according to the guest observer facility recommended criteria, and limited to the unit PCU2 because of known background control issues with unit PCU0. Although the RXTE monitoring of BL Lac did not occur on a daily basis, a light curve is derived 


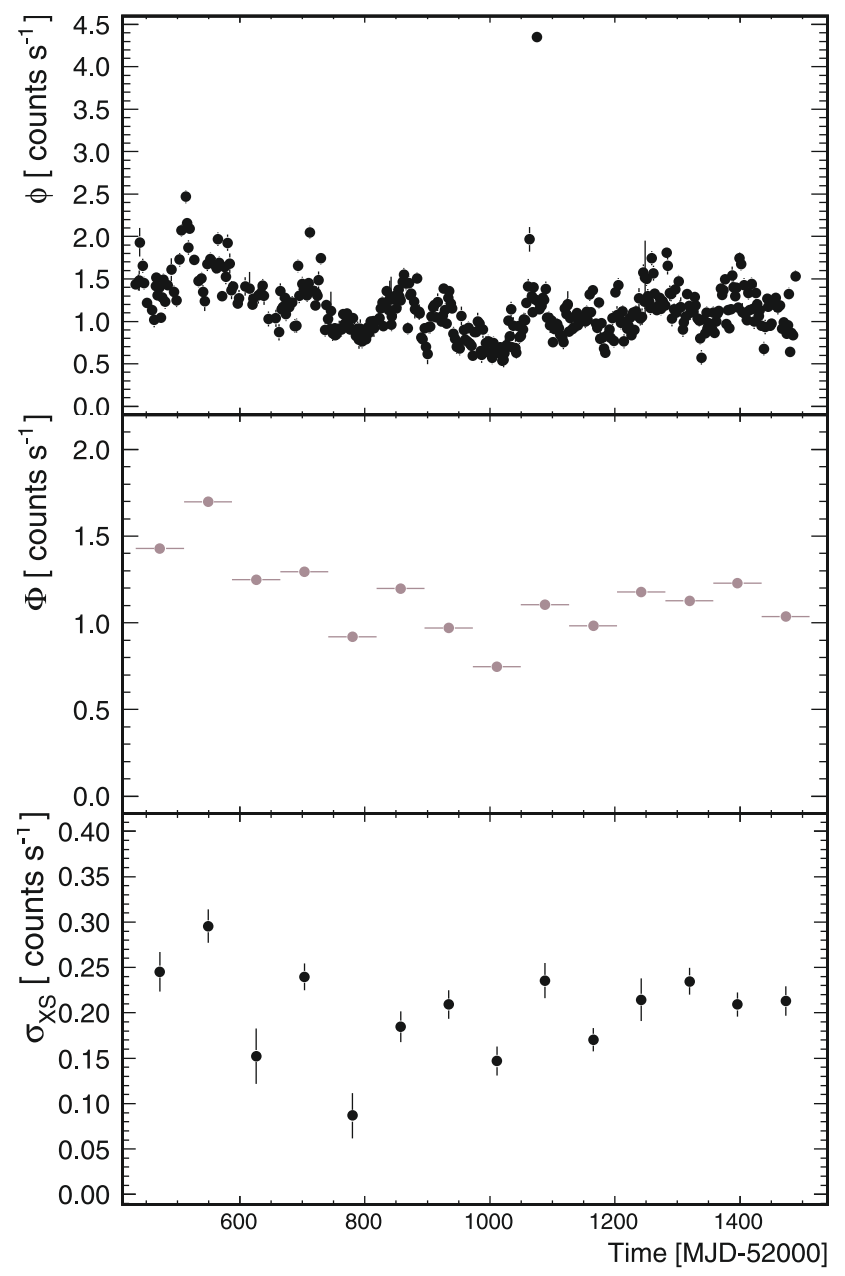

Fig. 1. Top panel: PCA flux light curve (in units of counts $\mathrm{s}^{-1}$ ) of BL Lac with bins of 1 day. Middle and bottom panels: PCA mean flux and excess rms measured from 77 day segments (see text).

in 1 day bins (top panel in Fig. 1), yielding 402 flux estimates $\phi_{i}$ (and associated measurement errors $\sigma_{i}$ ). In order to apply Gaussian statistics to our variance measurements, this light curve is divided further into segments of equal length such that each contain at least $N=20$ points (middle panel) of arithmetic mean $\Phi$. The shortest timescale allowing this condition is found to be $T=77 \mathrm{~d}$, where most of the segments have $N>20$ flux points. The excess rms $\sigma_{\mathrm{XS}}$, defined as $\sigma_{\mathrm{XS}}^{2}=\frac{1}{N} \sum_{i=1}^{i=N}\left[\left(\phi_{i}-\Phi\right)^{2}-\sigma_{i}^{2}\right]$ (Vaughan et al. 2003), which estimates the rms corrected for the Poisson noise, is then derived for each of the segments (bottom panel). Varying this length $T$, or requiring a larger amount of points $N$ in the variance estimation, does not change significantly the results presented here. The error in the excess variance is the $68 \%$ confidence level estimated from $10^{4}$ simulated light curves, where a random flux from a Gaussian of dispersion equal to the flux error $\sigma_{i}$ is added to each individual flux estimate $\phi_{i}$ (as in Appendix B in Vaughan et al. 2003). Its relatively flat light curve compared to other X-ray blazars, along with the highest flux point visible at MJD 53075, where the flux more than tripled, indicates that the source spends most of its time in a nonflaring state, which we refer to as its "quiescent" state. The short forementioned flare is clearly an exception in the overall variability of this source. A more detailed analysis of this single $2.4 \mathrm{ks}$ observation shows that the $2-10 \mathrm{keV}$ flux of $5.2 \times 10^{-11} \mathrm{erg} \mathrm{cm}^{-2} \mathrm{~s}^{-1}$ was associated with a spectral power law index of $\Gamma=2.5 \pm 0.1$,
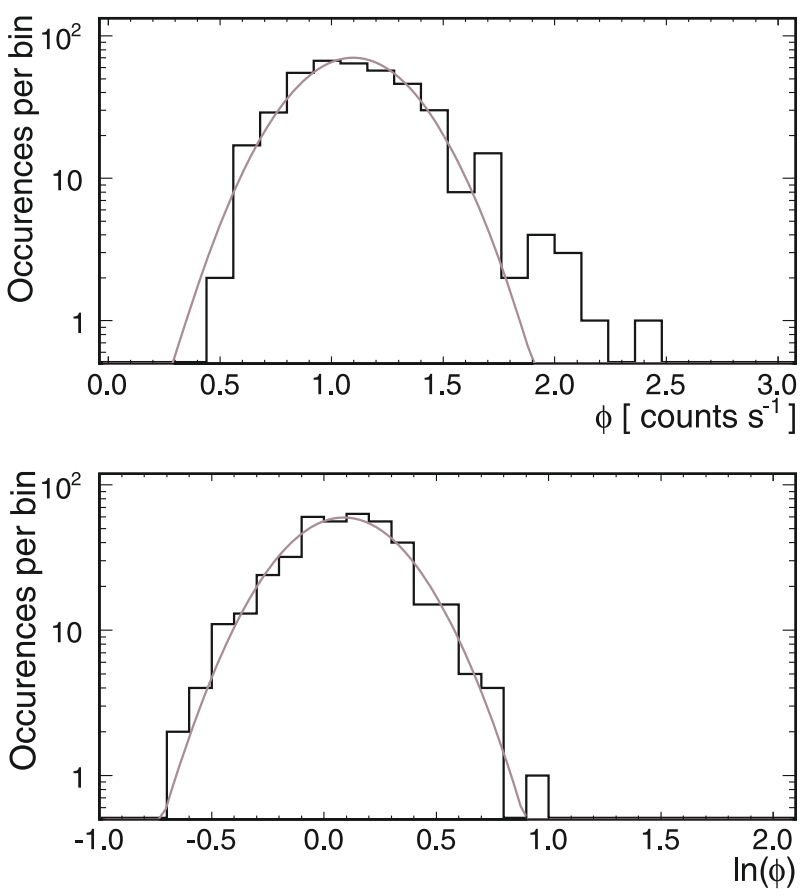

Fig. 2. Distribution of the fluxes for the considered period (top) and the distribution of the logarithm of the fluxes (bottom). The lines are the result of a Gaussian fit to the data. The improvement to the fit provided by the logarithmic function is clearly apparent in that it reproduces more successfully the steeper-than-Gaussian left-hand part of the distribution, as well as the broader, tail-like right hand part, which are characteristic of the lognormal distribution. The single flux estimation of the flare on MJD 54075 has been excluded so as not to favour the logarithmic fit.

much brighter than the $1-3 \times 10^{-11} \mathrm{erg} \mathrm{cm}^{-2} \mathrm{~s}^{-1}$ fluxes reported in Fig. 2 of Marscher et al. (2008), but also very soft compared to its brightness. Since our aim is to characterize the long-term lightcurve, this single observation is excluded from the analysis, removing one flux estimation out of 402 . It becomes obvious why removing this event is also conservative in terms of establishing the nature of the variability.

The light curve fluxes are presented in a histogram in Fig. 2 (top panel), along with the result of a chi-square Gaussian fit. The fit of the distribution of the fluxes has a chi-square probability of $p\left(\chi^{2}\right)=0.015\left(\chi^{2}=26.4\right.$, n.d.f $\left.=13\right)$, and a width of $\sigma=0.26 \mathrm{~s}^{-1}$, indicating that statistical fluctuations alone cannot explain the fit. The natural logarithm of the fluxes provide a much better Gaussian fit with $p\left(\chi^{2}\right)=0.47\left(\chi^{2}=12.75\right.$, n.d.f. $=13$ ) (bottom panel), and a width of $\sigma=0.26$. The scatter plot of $\sigma_{\mathrm{XS}}$ versus the average flux in Fig. 3, in which the short flare is excluded, shows a clear correlation $(\rho=0.73)$. A fit to a line $\left(\chi^{2}=33\right.$, n.d.f $\left.=12\right)$ yields $\overline{\sigma_{\mathrm{XS}}} \propto(0.15 \pm 0.02) \Phi$. An F-test shows that the improvement to a line fit is significant at a confidence level exceeding 99\% compared to a constant fit $\left(\chi^{2}=85\right.$, n.d.f $=13$ ). Including the flare observation causes the linear fit to be further preferred to the constant fit, and increases the slope of the linear fit by $\sim 4 \%$. It is important to remark that the flare observation is statistically very unlikely to be just a large variation of the same lognormal process, which is another indication that it is more likely to be a physically distinct state. 


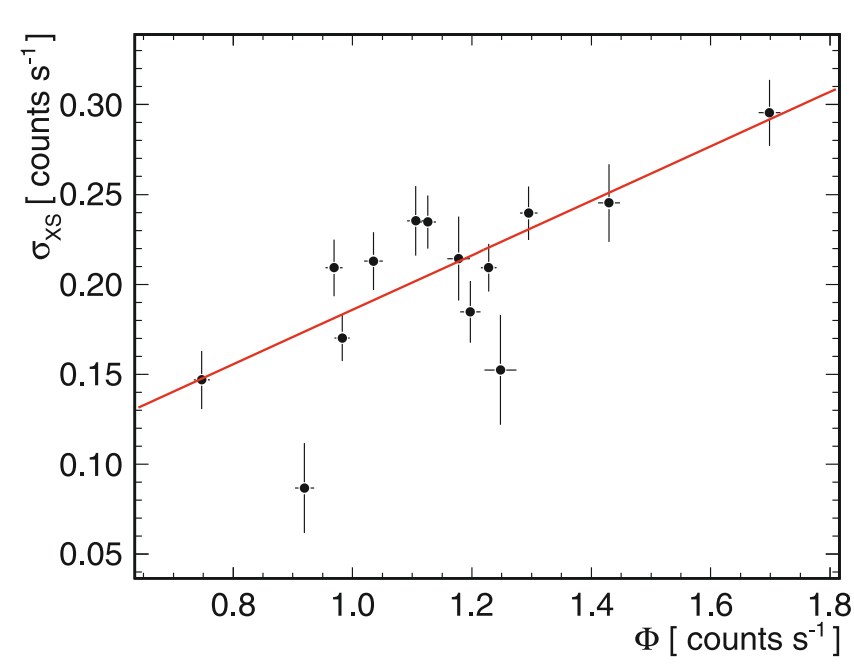

Fig. 3. Scatter plot of the excess variance versus the average of the fluxes for which the excess was determined. The line is a linear fit showing $\sigma_{\mathrm{XS}} \propto(0.15 \pm 0.02) \Phi$.

\section{Discussion}

The lognormality of the flux distributions, and the evidence that the fluctuations in the flux are proportional to the flux, imply that the variations are lognormal (Aitchinson et al. 1963). The proportionality factor of 0.15 in Fig. 3 is comparable to that (0.17 with a 1 -day mean flux binning using ROSAT data, and 0.26 with a 0.5 -day binning using $A S C A$ data) found in the most X-ray variable narrow-line Seyfert 1 galaxy IRAS 13 244-3809 by Gaskell (2004), the only other known case of an extragalactic source exhibiting lognormal X-ray flux variations. The width in Fig. 2 of $\sigma=0.26$ is however smaller ( 0.44 dex and 0.54 dex for the two different data sets mentioned above) than in IRAS 13244-3809, meaning that the fluctuations are smaller in this BL Lac than in the Seyfert galaxy. A linear correlation between the excess rms and the average flux was also found in the Seyfert 1 galaxy Mrk 766 (Vaughan et al. 2003). The physical process responsible for X-ray emission in Seyfert 1 galaxies is probably thermal emission from the accretion disk. We also note that a linear flux-rms relationship was found for Galactic accreting sources such as X-ray binaries Cyg X-1 and SAX J 1808.4-3658 by Uttley \& McHardy (2001), of proportionality factors of 0.33 and 0.31 , respectively. A specific observation of Cyg X-1 exhibited a remarkable lognormal fit to its X-ray flux distribution (Uttley et al. 2005). The origin of the linear flux-rms was interpreted by Uttley \& McHardy (2001) as originating from the subdivision of magnetic reconnection energy release as an avalanche occuring on large scales in the corona. The same authors note that the radius-dependent fluctuations in the mass accretion rate in the model of Lyubarskii et al. (1997) can also explain this relationship. It seems possible that the photon breeding mechanism (Stern \& Poutanen 2008, and references therein), which generates highly non-linear light curves in relativistic jets and operates in sites that are not necessarily directly connected to accretion properties, could be an alternative explanation of lognormal blazar variability, but this remains to be investigated since the statistical properties of the photon luminosity from this model are not known at this point.

These striking similarities suggest that the variability mechanism causing the X-ray modulation in BL Lac, outside of flaring events, is independent of the emission mechanism, and originates in the disk, rather than in the jet, provided these lognormal fluctuations are indeed present in the disk. The nonthermal emission from BL Lac is usually modeled by the synchrotron self-Compton scenario (SSC, see e.g., Maraschi et al. 1992; Bicknell \& Wagner 2002), where relativistic electrons cool either via the synchrotron or the inverse Compton mechanism. The comptonized photons are assumed to be predominantly the synchrotron photons themselves, rather than photons coming from the outside the emission region as in the external Compton model (EC, see e.g. Sikora et al. 1994), given the absence of thermal signatures in the emission spectrum of BL Lac. However, these mechanisms do not provide a way to generate lognormal variability if one of the parameters affecting the predicted flux (e.g., magnetic field, Doppler factor, injection rate) does not have a lognormal time-dependence itself. If fluctuations in the disk are indeed the origin of the lognormal radiation in BL Lac, then the most appropriate hypothesis would be one in which lognormal fluctuations in the accretion rate yield an injection rate with similar properties. If however external photons are at least partly responsible for the cooling of the X-ray emitting electrons, then lognormal fluctuations in the disk luminosity would have a similar result. An ambitious observation programme targeting other X-ray bright blazars, over similar time scales, and with a similar sampling quality, would allow us to search for lognormal variability, and perhaps solve an important piece in the puzzle about blazar variability.

Acknowledgements. We thank the anonymous referee for the careful reading and the constructive remarks, which have improved this report. B.G. gratefully acknowledges comments from D. Barret and G. Dubus on an early draft.

\section{References}

Albert, J., Aliu, E., Anderhub, H., et al. 2007, ApJ, 666, 17

Aitchinson, J., \& Brown, J. A. C. 1963, The Lognormal Distribution (Cambridge: Cambridge Press)

Bicknell, G. V., \& Wagner, S. J. 2002, PASA, 19, 129

Degrange, B., Superina, G., Giebels, B., et al. 2008, The emission of blazars in VHE gamma-rays viewed as a random stationary process: the case of PKS 2155-304, in Proceedings of Workshop on Blazar Variability across the Electromagnetic Spectrum, PoS(BLAZARS2008)016

Gaskell, M. G. 2004, ApJ, 612, L61

Jahoda, K., Swank, J. H., Giles, A. B., et al. 1996, Proc. SPIE, 2808, 59

Lyubaskii, Y. E. 1997, MNRAS, 292, 679

McHardy, I., Explaining X-ray Variability in Blazars, in Workshop on Blazar Variability across the Electromagnetic Spectrum, PoS(BLAZARS2008)014 Maraschi, L., Ghisellini, G., \& Celotti, A. 1992, ApJ, 397, L5

Marscher, A. P., Jorstad, S. G., D’Arcangelo, F. D., et al. 2008, Nature, 452, 966 Perlman, E. S., Addison, B., et al., Thermal AGN signatures in blazars, in Workshop on Blazar Variability across the Electromagnetic Spectrum, PoS(BLAZARS2008)009

Sikora, M., Begelman, M. C., \& Rees, M. J. 1994, ApJ, 421,153

Stern, B. E., \& Poutanen, J. 2008, MNRAS, 383, 1695

Uttley, P., \& McHardy, I. M. 2001, MNRAS, 323, L26

Uttley, P., McHardy, I. M., \& Vaughan, S. 2005, MNRAS, 359, 345

Vaughan, S., Edelson, R., Warwick, R. S., \& Uttley, P. 2003, MNRAS, 345, 1271 